\title{
Validation of treatment algorithm based on the Japan narrow-band imaging expert team classification for sub-centimeter colorectal polyps
}

\section{(ㄷ)(ㅇ) $\Theta$}

\author{
Authors \\ Kikuchi', Junichi Shiraishi ${ }^{2}$, Toshio Uraoka' ${ }^{1}$ \\ Institutions \\ 1 Department of Gastroenterology, National Hospital \\ Organization Tokyo Medical Center, Tokyo, Japan \\ 2 Department of Pathology, National Hospital \\ Organization Tokyo Medical Center, Tokyo, Japan
}

Motohiko Kato ${ }^{1}$, Keiichiro Abe ${ }^{1}$, Yoko Kubosawa ${ }^{1}$, Yukie Sunata ${ }^{1}$, Yuichiro Hirai ${ }^{1}$, Tetsu Hirata ${ }^{1}$, Yoshiaki Takada ${ }^{1}$, Michiko Wada ${ }^{1}$, Yusaku Takatori ${ }^{1}$, Shigeo Banno ${ }^{1}$, Satoshi Kinoshita ${ }^{1}$, Hideki Mori ${ }^{1}$, Kaoru Takabayashi ${ }^{1}$, Masahiro

submitted 18.12 .2017

accepted after revision 9.4.2018

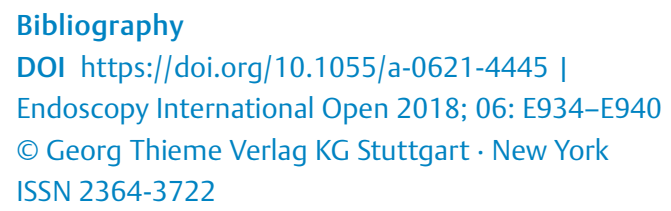

Corresponding author

Motohiko Kato, MD, PhD, Department of Gastroenterology, National Hospital Organization Tokyo Medical Center, 2-5-1 Higashigaoka, Meguro-ku, Tokyo, 152-8902, Japan

Fax: +81-3-3412-9811

moto28hiko@icloud.com

\section{ABSTRACT}

Background and study aims Although cold polypectomy $(C P)$ is widely used for colorectal polyps $<10 \mathrm{~mm}$, appropriateness of indications for $\mathrm{CP}$ or endoscopic mucosal resection (EMR) are still unclear. The aim of this study was to va- lidate the endoscopic treatment algorithm based on the Japan NBI Expert Team (JNET) classification.

Patients and methods Consecutive patients with at least one colorectal non-pedunculated polyp $<10 \mathrm{~mm}$ between July 2014 and October 2016 were included in this retrospective study. During the period, EMR was performed for JNET $\geq 2 B$ lesions and $C P$ for JNET $<2 A$. Among a total of 3966 lesions, 3368 lesions with JNET $\leq 2 \mathrm{~A}$ were resected by $\mathrm{CP}$ in compliance with the treatment algorithm but 565 resections for JNET $\leq 2 \mathrm{~A}$ were not compliant (by EMR), while all 24 JNET > 2B lesions were removed by EMR in compliance with the algorithm. Polypectomy outcomes were compared between the compliant and non-compliant groups. Histological outcomes were analyzed in accordance with JNET classification.

Results Post-polypectomy bleeding rate in the compliant group ( $0 \%$ ) was lower than that in the non-compliant group $(0.53 \%, P<0.01)$. Proportion of lesions diagnosed as cancer ( $38 \%$ vs $0.36 \%, P<0.01$ ) or submucosal cancer $(4.2 \%$ vs $0.03 \%, P=0.012$ ), and the lesion with free resection margin ( $91 \%$ vs $64 \%, P<0.01$ ) was higher in the JNET $\geq 2 B$ than JNET $\leq 2 \mathrm{~A}$.

Conclusion This study indicated our algorithm would be valid: $C P$ is suitable for most polyps $<10 \mathrm{~mm}$ as incidence of post-polypectomy bleeding is low, whereas EMR is recommended for JNET $\geq 2$ B lesions for histological complete removal.

\section{Introduction}

Colorectal cancer is the third most common cancer in the world, contributing to $9.7 \%$ (1.4 million) of the total number of new cases diagnosed in 2012 [1]. Colorectal polyps are considered to be precursors to colorectal cancer because cancer arises from adenomas by accumulation of mutations of cancer suppressor genes in a process known as the adenoma-carcinoma sequence [2].
Both polypectomy using an electrocautery snare (hot snare polypectomy: HSP) and endoscopic mucosal resection (EMR) combined with submucosal injection $[3,4]$ were first reported in 1973. These endoscopic polypectomy methods have been widely used, and it has been reported that endoscopic polypectomy significantly reduces incidence and mortality of colorectal cancer [5, 6]. However, as the opportunity for endoscopic treatment has increased, complications have also increased, including delayed bleeding, perforation and post-polypectomy coag- 
ulation syndrome, [7] which are caused by excessive energization.

Cold polypectomy (CP) is a type of polypectomy in which polyps are physically cut without electric current. This technique was reported by Woods in 1989 as a method of eradicating diminutive polyps using biopsy forceps, [8] and Draganov reported that higher rates of complete resection were achieved using jumbo biopsy forceps [9]. This CP procedure using forceps is called cold forceps polypectomy (CFP). Moreover, Tappero first reported CP using a snare (called cold snare polypectomy: (SP) in 1992 [10].

Given its safety and convenience, use of CP has spread and it has been accepted as a treatment method for all subcentimeter lesions [9,11-14]. However, conventional HSP and EMR still have a role because they have a potential advantage in eradicating neoplastic lesions using electrocautery. Therefore, if patients are appropriately chosen for CP and EMR appropriately, outcomes of polypectomy could be improved. Magnified endoscopy with narrow band imaging (NBI-ME) has been reported to be useful for differentiating between adenoma and non-neoplastic lesions by estimating the depth of invasion of cancer [15-19]. In 2014, a universal consensus was made on NBI-ME diagnosis, and the Japan NBI Expert Team (JNET) classification was established in which the findings of NBI-ME are categorized into four subgroups and JNET 2B and 3 are findings indicative of cancer [20]. We have established a treatment algorithm in which CP is applied to lesions with JNET 1 or $2 \mathrm{~A}$, and EMR/ HSP is used for lesions with JNET 2B. Therefore, the aim of this study was to validate the appropriateness of our algorithm and to clarify the appropriate treatment strategy for polyps less than $10 \mathrm{~mm}$.

\section{Patients and methods}

\section{Study design}

This was a retrospective study in a tertiary hospital. Consecutive patients who underwent a colonoscopy in whom at least one colorectal polyp was detected in our department from july 2014 to October 2016 were included in this study. A total of 4668 lesions were endoscopically resected in our department during the study period. Of these, 607 lesions were excluded because their size exceeded $10 \mathrm{~mm}$ and 95 lesions were excluded because of pedunculated morphology. As a result, 3966 lesions were included in the data analysis. The analysis was performed on a per-lesion basis, since the outcomes of polypectomy is strongly associated with the properties of a lesion rather than those of a patient. Written informed consent was obtained from each patient included in the study. The study protocol conformed to the ethical guidelines of the 1975 Declaration of Helsinki as reflected in a prior approval by the institution's human research committee and approved by Tokyo medical center institutional review board on September 27th 2016.

\section{Colonoscopy procedure}

All endoscopists participated in an intensive, 1-hour, interactive training program on endoscopic diagnosis of colorectal polyp including diagnosis of NBI with magnification by an expert endoscopist (T.U.) before performing CP. Patients underwent bowel preparation consisting of sodium picosulfate the day before colonoscopy and 2 to $3 \mathrm{~L}$ of polyethylene glycol solution the morning before the procedure. Colonoscopies were exclusively performed using a colonoscope with magnification capability and water irrigation function (PCF-Q260AZI or PCFH290AZI, Olympus Co., Tokyo, Japan). After cecal intubation, all detected polyps were photographed, and their characteristics, including size, location, macroscopic type and findings of magnified endoscopy with NBI-ME, were documented. Lesion size was estimated according to comparison to endoscopic devices. Cessation of antithrombotic drugs was done in accordance with guidelines for gastroenterological endoscopy in patients undergoing antithrombotic treatment published by the Japan Gastroenterological Endoscopy Society in 2014 [21]. Procedures were performed by three experts who had performed more than 5000 colonoscopies, four gastroenterologists who had performed 1000 to 4999 colonoscopies and six trainees who had performed fewer than 1000 colonoscopies.

\section{Endoscopic diagnosis}

According to macroscopic findings, lesions were classified as pedunculated (0-Ip), sessile (0-Is, Isp), flat ( 0 -IIa) or depressed (0-Ilc, $0-I l a+I l c, 0-I s+\| c)$ according to the Paris classification [22]. Differentiation among non-neoplastic lesions, adenoma or cancer by macroscopic findings was made based on lesion shape and color. Hyperplastic polyps usually presented as whitish, flat, elevated lesions; adenoma exhibited a reddish color and irregular shape, and obvious depression and remarkable redness were findings suggestive of cancer. Following conventional white light imaging (WLI), we applied NBI-ME. The NBIME diagnosis was made according to the JNET classification, which is a universal diagnostic classification consisting of both microstructural and micro-vessel findings that was proposed in June 2014 [20].

\section{Treatment strategy based on endoscopic diagnosis}

If any lesion was found during colonoscopy, each endoscopist immediately decided whether to remove it based on real-time diagnosis including NBI-ME. All lesions suspected as neoplasms were resected. Each endoscopist chose the type of procedure for polypectomy according to macroscopic features or endoscopic diagnosis. The algorithm for the treatment strategy is shown in > Fig. 1.

Pedunculated polyps (Paris type 0 -Ip) were resected by HSP or EMR, and CP was not attempted due to the possibility of immediate or post-procedure bleeding. The treatment choice for other polypoid lesions (Paris type 0 -Is or 0 -Isp) and flat, elevated polyps (Paris type 0 -Ila) was determined according to NBIME findings. For JNET Type I polyps, those larger than $5 \mathrm{~mm}$ located on the right side of the colon were resected by CP because those findings suggested sessile serrated adenomas/ polyps. JNET Type 2A polyps were considered low-grade adenomas and they were treated with CP. We chose CSP or CFP according to polyp size; CSP was applied to small (6-9mm) polyps, and CFP was applied to diminutive $(1-5 \mathrm{~mm})$ polyps. As JNET Type 2B polyps were likely to be high-grade adenoma 


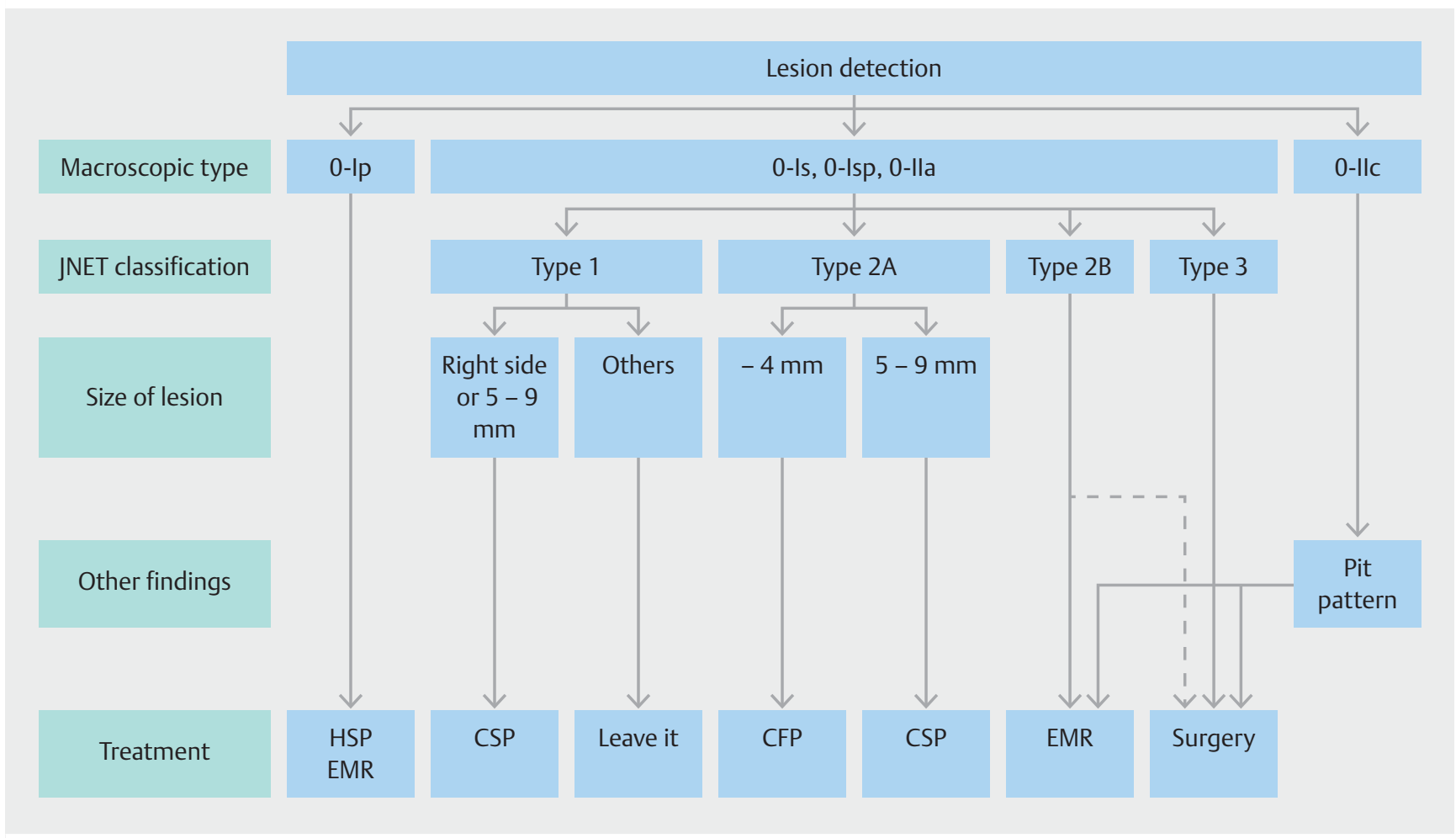

- Fig. 1 Treatment strategy algorithm.

or intramucosal cancer, they were treated with EMR unless there was an obvious finding of massive submucosal invasion. JNET Type 3 polyps were surgically resected because they are likely to be invasive cancers. Depressed lesions were resected by EMR as long as invasive signs were not present on pit pattern diagnosis using crystal violet staining.

\section{Cold polypectomy procedure}

In CSP, a polyp with a rim of surrounding normal mucosa was ensnared with a snare (Captivator II $10 \mathrm{~mm}$, Boston Scientific, Marlborough, Massachusetts, United States) and then removed by forced transection without use of electrocautery. In CFP, the polyp was removed with jumbo biopsy forceps (Radial Jaw 4 Jumbo Biopsy Forceps; Boston Scientific). After polypectomy, we created submucosal tamponade with forced water irrigation and confirmed that no residual tumor was present in post-polypectomy defects using NBI with magnification. Hemoclips were placed only in cases of continuous arterial bleeding immediately after $C P$.

\section{Outcome measurements}

To validate our algorithm of treatment strategy for colorectal polyps, we divided all lesions into two groups, compliant and non-compliant, according to whether they were resected in compliance with the algorithm from the viewpoint of the choice of either HSP/EMR or CP. A lesion was categorized as compliant if it was Paris type 0 -Ip or with JNET 2B/3 and resected by HSP/EMR or if was another type of lesion and was resected with CP. In this study, we didn't distinguish compliance based on $f$ the choice of either CFP or CSP. We compared clinical characteristics, outcomes of polypectomy and pathological findings between the compliant and non-compliant group. In addition, we compared pathological findings between the JNET 1, 2A (JNET $\leq 2 \mathrm{~A}$ ) group and JNET 2B, 3 (JNET $\geq 2 \mathrm{~B}$ ) group.

Location, macroscopic type, size, endoscopic diagnosis of WLI and NBI-ME diagnosis were noted as clinical characteristics. Macroscopic type was classified as polypoid (0-Is, 0 -Isp or 0 -Ip of the Paris classification) or flat (0-Ila or 0-Ilc of the Paris classification) [22]. A single endoscopist (K. A.) who was blinded to the pathological diagnosis reviewed the macroscopic type, WLI diagnosis and NBI-ME diagnosis using the recorded images. Location and size of lesions were referenced in the endoscopic record.

For polypectomy outcomes, we collected data concerning the proportion resected in a single piece, perforation and post-procedure bleeding. Bleeding was defined as an episode of obvious hematochezia with active bleeding or adherent clots on the post-polypectomy site confirmed by colonoscopy.

Pathological diagnosis was made based on the Vienna classification [23]. We assessed the cancer-bearing rate, proportion of submucosal cancer and proportion of negative margins as pathological findings. The cancer-bearing rate was defined as the proportion of lesions pathologically diagnosed as cancer to all resected lesions. A negative resected margin was limited to only lesions that were resected in a single piece with a tumorfree margin, and the outcome was treated as a non-negative margin when the lesion was lost or fragmented during retrieval. 


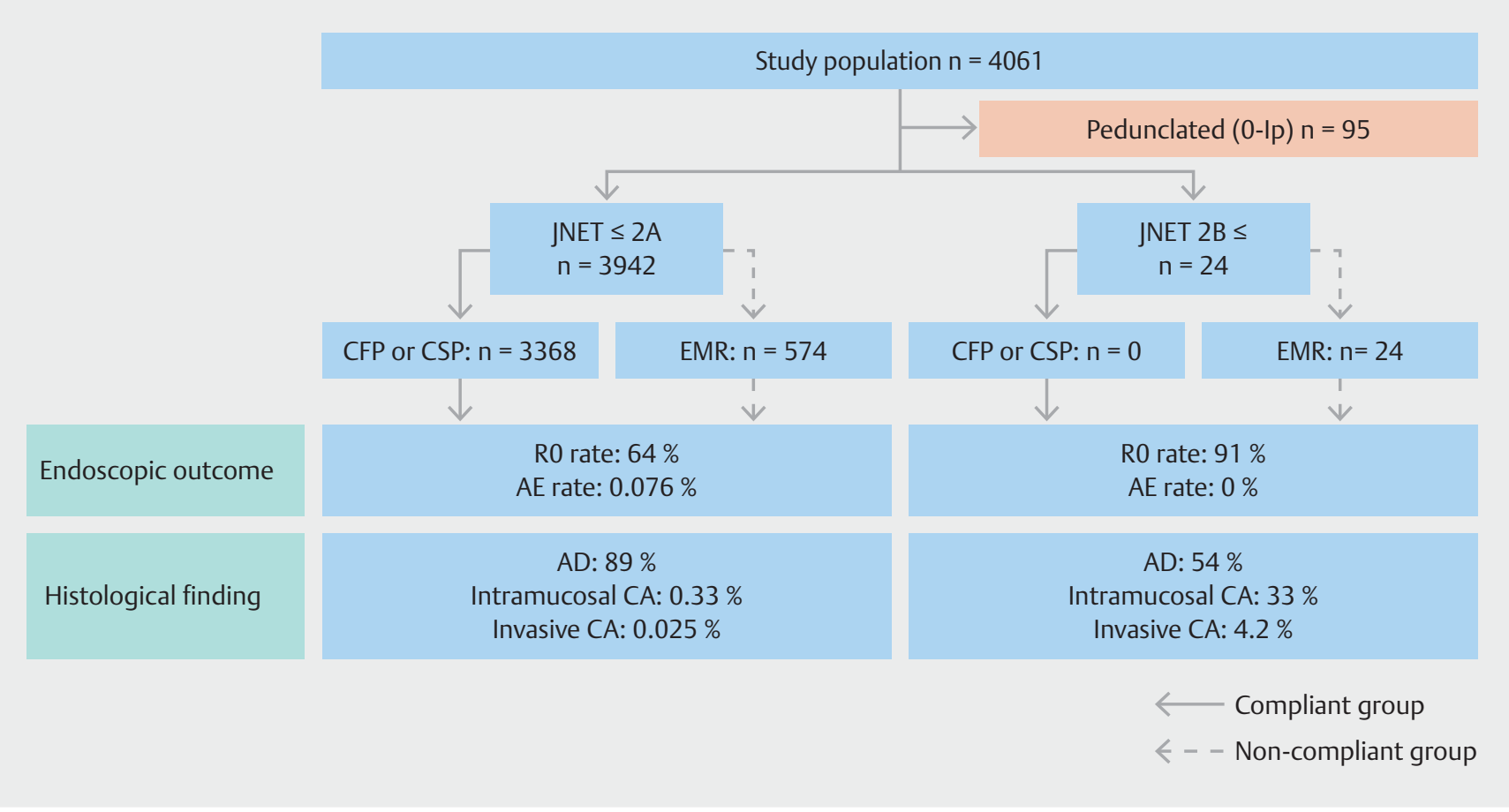

- Fig. 2 Flow diagram for lesions $<9 \mathrm{~mm}$.

The protocol compliance rate was defined as the proportion of polyps subjected to a polypectomy method prescribed by the protocol relative to all polyps. Moreover, the protocol compliance rate was analyzed by dividing the polypectomy method into HSP/EMR and CP groups.

\section{Statistical analysis}

Wilcoxon's rank sum test and Fisher's exact test were used to compare continuous and categorized factors. Outcomes of polypectomy are presented as percentages with $95 \%$ confidence intervals. Statistical significance was defined as $P<0.05$. Data analysis was performed using JMP software (ver. 11.1.1, SAS Institute Inc., Cary, North Carolina, United States).

\section{Results}

\section{Clinical characteristics}

A flow diagram of included lesions is shown in $>$ Fig. 2. The compliant group included 3401 lesions and the non-compliant group included 565 lesions. The proportion of lesions resected in compliance with the algorithm was $85.8 \%$. The most common reason for violation was application of EMR/HSP for lesions with JNET $\leq 2 \mathrm{~A}$ (99.7\%). The clinical characteristics of the examined lesions are described in $>$ Table 1 . Approximately $60 \%$ of lesions were located in the right colon. Over $90 \%$ of the lesions had polypoid morphology. Mean polyp size was $4.6 \pm 1.7 \mathrm{~mm}$. Approximately $99 \%$ of lesions were classified as JNET $2 \mathrm{~A}$. When the study population was divided into the compliant and noncompliant groups, significantly more lesions were located on the right side and lesion size was significantly smaller in the compliant group. The main reason for violating the algorithm
- Table 1 Clinical characteristic of the lesions $(n=3966)$.

\begin{tabular}{|l|l|r|}
\hline Location & Cecum & $7 \%$ \\
& Ascending colon & $24 \%$ \\
\hline & Transverse colon & $26 \%$ \\
\hline & Descending colon & $9 \%$ \\
\hline & Sigmoid colon & $24 \%$ \\
\hline Rectum & $10 \%$ \\
\hline Macroscopic type & Sessile & $93 \%$ \\
\hline Size & Flat & $6 \%$ \\
\hline Japan NBI Expert & Depressed & $1 \%$ \\
\hline classification & Mean \pm SD & $4.6 \pm$ SD1.7 mm \\
\hline & 1 & $1.7 \%$ \\
\hline & 2A & $97.7 \%$ \\
\hline & $2 B$ & $0.6 \%$ \\
\hline
\end{tabular}

was application of EMR/HSP for JNET $\leq 2 \mathrm{~A}$ and the majority of the non-compliant group was resected with EMR/HSP ( $>$ Table2).

\section{Outcomes of polypectomy in compliant and non- compliant group}

Outcomes of endoscopic treatment in both the compliant and non-compliant groups are described in $\downarrow$ Table 3 . Resection in a single piece was achieved in more than $97 \%$ of lesions in both groups. Perforation did not occur in either group. Delayed bleeding occurred only in the non-compliant group, and there was a significant difference in the proportion of lesions with delayed bleeding. 
- Table 2 Characteristics of lesions in compliant and non-compliant groups.

\begin{tabular}{|c|c|c|c|c|}
\hline Factors & & $\begin{array}{l}\text { Compliant } \\
(\mathrm{N}=3401)\end{array}$ & $\begin{array}{l}\text { Non-compliant } \\
(\mathrm{N}=565)\end{array}$ & $P$ value \\
\hline Location & Right side & $60 \%$ & $47 \%$ & $<0.01^{*}$ \\
\hline Macroscopic type & Polypoid & $93 \%$ & $93 \%$ & 0.98 \\
\hline Size & Median [range] & $4[2-9] \mathrm{mm}$ & $7[2-9] \mathrm{mm}$ & $<0.01^{*}$ \\
\hline WLI diagnosis & Adenoma & $98 \%$ & $95 \%$ & $<0.01^{*}$ \\
\hline JNET classification & $\begin{array}{l}\leq 2 \mathrm{~A} \\
\geq 2 \mathrm{~B}\end{array}$ & $\begin{array}{l}99.3 \% \\
0.68 \%\end{array}$ & $\begin{array}{l}99.8 \% \\
0.18 \%\end{array}$ & 0.24 \\
\hline Endoscopic treatment & $\begin{array}{l}\text { CP } \\
\text { EMR/HSP }\end{array}$ & $\begin{array}{r}99.0 \% \\
1.0 \%\end{array}$ & $\begin{array}{l}0.35 \% \\
99.6 \%\end{array}$ & $<0.01^{*}$ \\
\hline
\end{tabular}

- Table 3 Outcomes of endoscopic treatment in compliant and non-compliant groups.

\begin{tabular}{|l|l|l|l|}
\hline Factors & $\begin{array}{l}\text { Compliant } \\
\text { (N=3401) }\end{array}$ & $\begin{array}{l}\text { Non-compliant } \\
(\mathbf{N}=\mathbf{5 6 5})\end{array}$ & 0.068 \\
\hline $\begin{array}{l}\text { Resection in a single piece } \\
{[95 \% \mathrm{Cl}]}\end{array}$ & $98.2 \%$ & $97.0 \%$ & {$[95.2-98.1 \%]$} \\
\hline Perforation & {$[97.7-98.6 \%]$} & $0 \%$ \\
\hline$[95 \% \mathrm{Cl}]$ & $0 \%$ & {$[0-0.68 \%]$} & $<0.01^{*}$ \\
\hline Bleeding & {$[0-0.11 \%]$} & $0.53 \%$ & {$[0.18-1.5 \%]$} \\
\hline $95 \% \mathrm{Cl}]$ & $0 \%$ & & \\
\hline$*$ statistically significant & {$[0-0.11 \%]$} & & \\
\hline
\end{tabular}

- Table 4 Pathological findings of lesions $\leq$ JNET 2A and $\geq$ JNET 2B.

\begin{tabular}{|c|c|c|c|}
\hline Factors & $\leq$ JNET $2 A$ & $\geq$ JNET 2B & $P$ value \\
\hline $\begin{array}{l}\text { Cancer-bearing rate } \\
{[95 \% \mathrm{Cl}]}\end{array}$ & $\begin{array}{l}0.36 \% \\
{[0.21-0.60 \%]}\end{array}$ & $\begin{array}{l}38 \% \\
{[21-57 \%]}\end{array}$ & $<0.01^{*}$ \\
\hline $\begin{array}{l}\text { Proportion of Submucosal cancer } \\
{[95 \% \mathrm{Cl}]}\end{array}$ & $\begin{array}{l}0.03 \% \\
{[0.00-0.14 \%]}\end{array}$ & $\begin{array}{l}4.2 \% \\
{[0.7-20 \%]}\end{array}$ & $0.012^{*}$ \\
\hline $\begin{array}{l}\text { Negative margin } \\
{[95 \% \mathrm{Cl}]}\end{array}$ & $\begin{array}{l}64 \% \\
{[62-65 \%]}\end{array}$ & $\begin{array}{l}91 \% \\
{[72-97 \%]}\end{array}$ & $<0.01^{*}$ \\
\hline
\end{tabular}

\section{Comparison of pathological findings between JNET $\leq 2 \mathrm{~A}$ and $\geq 2 \mathrm{~B}$ group}

Pathological findings of the resected specimens according to NBI-ME findings are shown in $>$ Table 4 . There were many more lesions diagnosed as cancer pathologically in the JNET $\geq 2 \mathrm{~B}$ group (38\%) than in the JNET $\leq 2 \mathrm{~A}$ group $(0.36 \%)$. Similarly, the proportion of submucosal cancer was much higher in the JNET $\geq 2 \mathrm{~B}$ group ( $4.2 \%$ vs $0.03 \%$ ). With regard to the incidence of negative resection margins, it was significantly higher in the compliant group ( $91 \%$ vs $64 \%$ ).

\section{Comparison of outcomes between CP and EMR/HSP}

Resection in a single piece was achieved in approximately $98 \%$ of lesions in both groups. Perforation did not occur in either group. Delayed bleeding occurred in three lesions $(0.50 \%, 95 \%$ $\mathrm{Cl} 0.17-1.5 \%$ ) resected by EMR/HSP, whereas no lesion treated with CP was associated with delayed bleeding. The cancer-bearing rate was approximately 10 -fold higher for EMR/HSP than for CP $(2.5 \%$ vs $0.24 \%, P<0.01)$. Submucosal cancer was found only in the EMR group. The incidence of negative resection margins was significantly higher in the EMR group ( $86 \%$ vs $60 \%, P<$ $0.01)$. 


\section{Discussion}

In this retrospective study, we analyzed 4061 endoscopically resected consecutive polyps. Post-polypectomy bleeding did not occur at all in 3401 polyps resected with CP, and the proportion of post-polypectomy bleeding of $\mathrm{CP}$ was significantly lower than that for HSP/EMR. However, the negative margin rate for CP was $60 \%$, which was significantly lower than that for HSP/ EMR. According to our treatment strategy algorithm, the cancer-bearing rate for HSP/EMR was 10-fold higher than that for CP.

Previous studies have reported that CP prevented post-polypectomy bleeding [8-10]. Recently, Horiuchi reported that CSP did not cause post-polypectomy bleeding in patients receiving anticoagulant treatment, and a significant difference was observed between CSP and EMR [13]. These results suggest that CP would reduce post-polypectomy bleeding as well as perforation or post-polypectomy electrocoagulation syndrome, although electrocoagulation is believed to prevent bleeding immediately after polypectomy. The results of our study showed that post-polypectomy bleeding was not found in more than 3000 consecutive polyps resected by CP, which provides evidence of the safety of CP.

Despite its safety, some concerns exist regarding $\mathrm{CP}$. One concern is the inferiority of the curability of polyps removed using CP. The negative margin rate for CP was only $60 \%$, which was significantly lower than that for HSP/EMR (approximately $85 \%$ in our study). Not using an electric current leads to fewer complications but increases the risk of local residue due to absence of a burning effect. Furthermore, maintaining sufficient margins is difficult in CP because a polyp cannot be physically removed, as opposed to EMR, if excess surrounding mucosa is captured. Another concern is the difficulty of pathological diagnosis of specimens resected by CP. Tutticci reported that resection depth with CSP was relatively shallow, with a shallow layer of submucosa and occasionally muscularis mucosa [24]. Specimens were thin and fragile and were therefore easily fragmented during retrieval by suctioning through the working channel of the colonoscope. It is also difficult to diagnose invasive cancer when a sufficient depth of the submucosal layer is not resected. Therefore, EMR/HSP is much better than CP in terms of clearness of margin resection and precise histological diagnosis.

According to previous reports, the proportion of invasive cancer in polyps smaller than $10 \mathrm{~mm}$ ranges from 0.2 to $0.9 \%$ $[25,26]$. The proportion of invasive cancer in our study was $0.27 \%$, which was consistent with previous studies. These proportions were low; however, they were not negligible when considering the disadvantage of $\mathrm{CP}$ mentioned above. Using macroscopic findings and the JNET classification, we established an algorithm for choosing the type of endoscopic treatment for small colorectal polyps. In this retrospective study, we analyzed 3966 endoscopically resected consecutive polyps to validate our algorithm for treatment strategy for small colorectal polyps. Post-polypectomy bleeding did not occur at all in 3401 polyps resected in compliance with our algorithm, and the proportion of post-polypectomy bleeding with CP was sig- nificantly lower than that in the non-compliant group. Moreover, both the cancer-bearing rate and proportion of submucosal cancer, and proportion of the lesion with pathological tumor-free margin were much higher in the JNET $\geq 2 \mathrm{~B}$ group. These results show our algorithm effectively picked up highrisk lesions and EMR/HSP (which is better in terms of clearness margin resection) or CP (which is better in terms of less complications) was appropriately chosen with significant decrease in complications.

The current study has several limitations due its single-center retrospective design. First, we analyzed only lesions that were endoscopically resected; therefore, we cannot eliminate a certain degree of selection bias. For example, small lesions surgically resected due to a suspicion of deep invasion were excluded from data analysis. Second, the compliance rate for treatment choices in this study was less than $50 \%$. However, concerning the choice between CP and EMR, the compliance rate was approximately $85 \%$. Although the results of our study should be interpreted carefully due to these limitations, our results, based on over 4000 polyps, indicate that CP should be considered as a treatment option.

\section{Conclusion}

In conclusion, this large-scale retrospective study demonstrated that our algorithm for treatment of small polyps based on endoscopic diagnosis, including NBI-ME, would be valid. Although CP is suitable for a large proportion of small polyps due to its safety, EMR should still be used for an accurate pathological diagnosis when polyps are suspected as cancer by NBIME.

\section{Competing interests}

None

\section{References}

[1] WHO. World Cancer Research Fund International. 2012

[2] Cho KR, Vogelstein B. Suppressor gene alterations in the colorectal adenoma-carcinoma sequence. Journal of cellular biochemistry Supplement 1992; 16G: 137-141

[3] Deyhle P. A method for endoscopic electroresection of sessile colonoc polyps. Endoscopy 1973; 5: 38-40

[4] Wolff WI, Shinya H. Polypectomy via the fiberoptic colonoscope. Removal of neoplasms beyond reach of the sigmoidoscope. N Engl J Med 1973; 288: 329-332

[5] Zauber AG, Winawer S], O'Brien M] et al. Colonoscopic polypectomy and long-term prevention of colorectal-cancer deaths. N Engl J Med 2012; 366: 687-696

[6] Atkin WS, Edwards R, Kralj-Hans I et al. Once-only flexible sigmoidoscopy screening in prevention of colorectal cancer: a multicentre randomised controlled trial. Lancet (London, England) 2010; 375: $1624-1633$

[7] Fisher DA, Maple JT. Committee ASoP. et al. Complications of colonoscopy. Gastrointest Endosc 2011; 74: $745-752$ 
[8] Woods A, Sanowski RA, Wadas DD et al. Eradication of diminutive polyps: a prospective evaluation of bipolar coagulation versus conventional biopsy removal. Gastrointest Endosc 1989; 35: 536 - 540

[9] Draganov PV, Chang MN, Alkhasawneh A et al. Randomized, controlled trial of standard, large-capacity versus jumbo biopsy forceps for polypectomy of small, sessile, colorectal polyps. Gastrointestinal Endoscopy 2012; 75: $118-126$

[10] Tappero G, Gaia E, De Giuli P et al. Cold snare excision of small colorectal polyps. Gastrointest Endosc 1992; 38: 310 - 313

[11] Raad D, Tripathi P, Cooper $G$ et al. Role of the cold biopsy technique in diminutive and small colonic polyp removal: a systematic review and meta-analysis. Gastrointest Endosc 2016; 83: 508 - 515

[12] Uraoka T, Ramberan H, Matsuda T et al. Cold polypectomy techniques for diminutive polyps in the colorectum. Dig Endosc 2014; 26: (Suppl. $02): 98-103$

[13] Horiuchi A, Nakayama Y, Kajiyama M et al. Removal of small colorectal polyps in anticoagulated patients: a prospective randomized comparison of cold snare and conventional polypectomy. Gastrointest Endosc 2014; 79: 417-423

[14] Repici A, Hassan C, Vitetta E et al. Safety of cold polypectomy for $<10 \mathrm{~mm}$ polyps at colonoscopy: a prospective multicenter study. Endoscopy 2011; 44: $27-31$

[15] Kanao H, Tanaka S, Oka S et al. Narrow-band imaging magnification predicts the histology and invasion depth of colorectal tumors. Gastrointest Endosc 2009; 69: 631-636

[16] Sano Y, Ikematsu H, Fu KI et al. Meshed capillary vessels by use of narrow-band imaging for differential diagnosis of small colorectal polyps. Gastrointest Endosc 2009; 69: $278-283$

[17] Wada Y, Kudo SE, Kashida $\mathrm{H}$ et al. Diagnosis of colorectal lesions with the magnifying narrow-band imaging system. Gastrointest Endosc 2009; 70: $522-531$
[18] Higashi R, Uraoka T, Kato J et al. Diagnostic accuracy of narrow-band imaging and pit pattern analysis significantly improved for less-experienced endoscopists after an expanded training program. Gastrointest Endosc 2010; 72: 127-135

[19] Ikematsu H, Matsuda T, Emura F et al. Efficacy of capillary pattern type IIIA/IIIB by magnifying narrow band imaging for estimating depth of invasion of early colorectal neoplasms. BMC gastroenterology 2010; 10: 33

[20] Sano Y, Tanaka S, Kudo SE et al. Narrow-band imaging (NBI) magnifying endoscopic classification of colorectal tumors proposed by the Japan NBI Expert Team. Dig Endosc 2016; 28: 526 - 533

[21] Fujimoto K, Fujishiro M, Kato M et al. Guidelines for gastroenterological endoscopy in patients undergoing antithrombotic treatment. Dig Endosc 2014; 26: 1 - 14

[22] [Anonymous] Update on the paris classification of superficial neoplastic lesions in the digestive tract. Endoscopy 2005; 37: 570- 578

[23] Stolte M. The new Vienna classification of epithelial neoplasia of the gastrointestinal tract: advantages and disadvantages. Virchows Arch 2003; 442: 99-106

[24] Tutticci N, Burgess NG, Pellise M et al. Characterization and significance of protrusions in the mucosal defect after cold snare polypectomy. Gastrointest Endosc 2015; 82: $523-528$

[25] Gschwantler M, Kriwanek S, Langner E et al. High-grade dysplasia and invasive carcinoma in colorectal adenomas: a multivariate analysis of the impact of adenoma and patient characteristics. Eur J Gastroenterol Hepatol 2002; 14: $183-188$

[26] Lieberman D, Moravec M, Holub J et al. Polyp size and advanced histology in patients undergoing colonoscopy screening: implications for CT colonography. Gastroenterology 2008; 135: 1100-1105 